\title{
Estrategias de reducción de riesgos con usuarios de drogas inyectadas: talleres de sexo más seguro
}

\author{
InsúA, P. ; MONCADA, S. ${ }^{2}$ \\ (1) Facultad de Psicología. Universidad del País Vasco. \\ (2) Plan Nacional sobre Drogas. \\ Enviar correspondencia:
}

Patricia Insúa. Facultad de Psicología. Universidad del País Vasco. Avda. Tolosa, 70. 20018 San Sebastián. E-mail: pbpincep@ss.ehu.es

\section{RESUMEN}

Objetivo: El objetivo es evaluar los resultados de, y la satisfacción de los usuarios con, 25 Talleres de sexo más seguro diseñados para reducir los riesgos asociados a la conducta sexual entre la población de usuarios de drogas inyectadas (UDIs).

Material y Método: La muestra incluye 387 UDIs (68,7\% hombres y $31,3 \%$ mujeres) con una edad promedio de 32,4 años, asignados de forma no aleatoria a los grupos de intervención y comparación. Los sujetos fueron evaluados en pre-test, post-test y seguimiento utilizando un cuestionario autoaplicado sobre conductas sexuales de riesgo y variables mediadoras en estas conductas.

Resultados: Los Talleres de sexo más seguro están asociados a un aumento en la frecuencia del uso del preservativo en las relaciones coitales, tanto con la pareja habitual como con pareja ocasional. También están asociados a un aumento en las variables mediadoras para las practicas de sexo seguro: conocimientos sobre el uso adecuado del preservativo, conocimientos sobre la transmisión del VIH/SIDA y expectativas de resultados del uso del preservativo. Adicionalmente, la satisfacción de los usuarios de estos Talleres es muy elevada.

Conclusión: Estos resultados proporcionan apoyo empírico a la eficacia de este tipo de intervenciones cortas diseñadas para reducir los riesgos asociados a las practicas sexuales entre los UDIs. Indirectamente proporcionan apoyo a la formación en redes realizada con profesionales sanitarios que trabajan en drogodependencias para fomentar las intervenciones de reducción de los riesgos entre sus pacientes UDIs.

Palabras clave: Programa Reducción de riesgos, UDIs, Conducta sexual, SIDA, Evaluación.

\section{ABSTRACT}

Objective: The objective is to evaluate the results and user satisfaction with 25 safer-sex workshops designed to reduce the risks associated with sexual behaviours among injecting drug users (IDUs).

Method: The sample includes 387 individuals $(68.7 \%$ male and $32.3 \%$ female IDUs) with an average of 32.4 years, assigned non-randomly to a comparison group or to a workshop user-group. They were assessed at pretest, postest and followup using a questionnaire that includes questions on high-risk sexual behaviours and mediating variables for these behaviours.

Results: Workshops designed for safer sex practices were associated with higher frequencies of condom use with a habitual or occasional partner. Also, these interventions were related to significant improvements in mediating variables for safer sex practices: knowledge on an adequate use of a preservative, knowledge on HIV and AIDS, and outcome expectancies about using a condom. Moreover, users of these workshops reported high satisfaction levels.

Conclusions: The findings provide encouraging support for the efficacy and user satisfaction of this type of short intervention, designed for risk-reduction associated with sex practices among injecting drug users. Indirectly, they provide support for training in networks, carried out by health professionals working in drug dependency to foster risk reduction interventions among their IDU patients.

Key words: Harm Reduction, IDUs, Tailored Programme, Sexual behaviour, AIDS, Evaluation.

\section{INTRODUCCIÓN}

E n nuestro país, los servicios de asistencia a drogodependientes se organizaron a finales de la década de los años 70, basados inicialmente en un modelo de la adicción como enfermedad orientado principalmente hacia la abstinencia en el uso de drogas. En los años 90, con la aparición de la infección por el Virus de la Inmunodeficiencia Humana (VIH) entre los inyectores de drogas y la consecuente epidemia de SIDA en este colectivo, surgieron los programas orientados hacia la reducción de los riesgos asociados a las conductas de uso de drogas. En España, en los últimos años, los distintos dispositivos de tratamiento (públicos y privados) han ido reconociendo la necesidad de diseñar e implementar programas 
para la prevención de los problemas de salud asociados al uso de drogas que se enmarquen en los modelos teóricos de reducción de riesgos especialmente con aquellos usuarios de drogas que utilizan, aunque sea de manera ocasional, la vía parenteral.

En los primeros momentos se cuestionó la eficacia de este tipo de programas, y las posibles consecuencias derivadas de objetivos que no perseguían la abstinencia. Sin embargo, numerosos estudios han demostrado ya su efectividad y eficiencia, su carácter no excluyente con otros Programas de tratamiento para las adicciones y la necesidad ineludible de que complementen a Programas destinados a conseguir la abstinencia.

La evaluación de los Programas de Intercambio de Jeringuillas (PIJs) han demostrado que están asociados a la reducción de las conductas de alto riesgo en el consumo de drogas por vía parenteral (Des Jarlais et al., 1999), y a la derivación de los usuarios a otros servicios de salud (Dolan, Stimson y Donoghoe, 1993). No obstante, diversas investigaciones indican que esta tendencia a adoptar conductas preventivas no se produce en las relaciones sexuales íntimas, y de forma particular con la pareja estable, y que los cambios en la conducta sexual (en caso de producirse) se dan de forma marcadamente más lenta que los cambios en la conducta de inyección y son menos estables y duraderos. Datos aportados por distintos autores (Battjes et al., 1995; Pavia et al., 1997; Menoyo et al., 1998) sugieren que la seguridad sexual no es considerada como una alta prioridad para los UDIs y sus parejas sexuales y si bien las normas cotidianas del grupo han comenzado a enfatizar el "uso seguro de drogas", enfatizan las prácticas sexuales inseguras

Numerosos estudios ya han demostrado que los UDIs son capaces de modificar sus conductas de riesgo en el uso inyectado de drogas de cara a reducirlos (Watters, Estilo, Kral et al...,1994; Watters, Estilo, Clark y Lorvick, 1994; Stimson y Power, 1992; Stimson, 1995). Sin embargo, los resultados de los estudios de evaluación sobre la prevención de la transmisión sexual del VIH/SIDA han sido menos prometedores (Dolezal et al., 1999) y han mostrado que las conductas sexuales de riesgo parecen ser más resistentes al cambio que las conductas de autoadministración de drogas. En general se acepta que facilitar la disponibilidad de preservativos es una condición necesaria pero no suficiente para reducir las conductas sexuales de riesgo y que es necesario implementar otro tipo de intervenciones educativas y preventivas, que hagan hincapié en aspectos motivacionales y actitudinales específicos, como son los "Talleres de Sexo Más Seguro" (Insúa,1996, Insúa et al., 1993; Becker, Rankin y Rickel, 1998). No obstante, la eficacia de estas intervenciones sobre las conductas de riesgo es relativamente menor que la de los programas de reducción de los riesgos asociados al uso inyectado de drogas, estimándose que el impacto de estos Talleres sobre el cambio de las conductas sexuales de riesgo se sitúa en torno a una reducción del 10-20\% en estas conductas.

Ante la necesidad de implementar programas específicos diseñados para la reducción de los riesgos asociados tanto a la conducta de inyección como a la conducta sexual, el Plan Nacional sobre Drogas, el Plan Nacional sobre el SIDA y la Universidad del País Vasco han puesto en marcha un Programa en Redes para la formación de profesionales sanitarios del Estado que trabajan en servicios públicos de atención a UDIs. El Programa se fundamenta en los principios de las intervenciones eficaces de salud pública para usuarios de drogas consensuados por la OMS y, tanto el diseño de la formación como las intervenciones concretas, se han realizado considerando los programas cuya evaluación ha demostrado que son eficaces para los objetivos que se proponen (Insúa y Moncada, 2000b; Insúa, Lledó y Grijalvo, 2001). Así, este Programa tiene sus fuentes teóricas en los modelos teóricos sociocognitivos que explican el cambio de comportamiento (el Modelo de la Acción Razonada (Ajzen y Fishbein, 1977; 1980); el Modelo de la Acción Planificada (Ajzen y Madden, 1986; Shifter y Ajzen, 1985); el Modelo de Creencias de Salud (Becker, 1974); el Modelo de Prevención de Recaídas (Gibbons, McGobern y Lando, 1991); el Modelo PRECEDE/PROCEED (Green y Kreuter, 1991); la Teoría de la Autoeficacia (Bandura, 1977); el Modelo de Fases de Cambio (Prochaska y DiClemente, 1983; 1992); el Modelo de Reducción de Riesgo de Sida (Catania et al., 1990) y el Modelo de Reducción de Riesgo de Sida Modificado (Ehrhardt et al., 1992)) y se consolida después de haber sido puesto a prueba y evaluado en distintas poblaciones de profesionales sanitarios del Estado durante los últimos años. Las evaluaciones de cada uno de los programas previamente realizados nos han permitido estructurar un programa de intervención en redes que incluye formación para los profesionales sanitarios en dos tipos de talleres: Talleres de Sexo más Seguro (TSMS) y Talleres de Consumo de menos Riesgo (TCMR), además de la supervisión para el diseño de intervenciones adaptadas al colectivo de UDIs en cada territorio y la facilitación de los materiales didácticos específicos: un Manual de Educación Sanitaria y dos vídeos psicoeducativos (Insúa, 1999), como materiales de apoyo. Este Programa ha sido descrito en profundidad en otros trabajos (Insúa y Moncada, 2000a; 2001).

En un informe previo (Insúa y Moncada, 2002) se presentaron resultados en apoyo a la eficacia de Talleres diseñados por los profesionales sanitarios de nuestro programa para la reducción de los riesgos asociados a la conducta de inyección de drogas en sus clientes UDIs. El presente informe describe los resultados alcanzados y la satisfacción de los usua- 
rios, en los talleres diseñados para fomentar el desarrollo de prácticas sexuales más seguras entre los UDIs en tratamiento por abuso de sustancias.

\section{MATERIALY MÉTODO}

\subsection{Descripción del Programa de intervención con usuarios de drogas orientado al desarrollo de prácticas sexuales más seguras}

El Programa consistió en desarrollar talleres de formación para la prevención de los problemas de salud asociados a la conducta sexual y específicamente para la prevención de la transmisión del VIH y otras enfermedades de transmisión sexual. Estas actividades estuvieron organizadas en forma de TSMSs, siguiendo los criterios de las intervenciones eficaces de salud pública, recogidos en otros trabajos nuestros (Insúa y Moncada, 2000b; Insúa, Lledó y Grijalvo, 2001).

El desarrollo de los TSMSs utilizó una metodología que combinó técnicas de dinámica de grupos para la participación de los usuarios, con la exposición semimagistral activa para la transmisión de los contenidos teóricos. La duración de los Talleres fue de 10 horas, distribuidas en cinco sesiones de dos horas cada una.

El objetivo general de este programa de intervención es la reducción de los riesgos y los daños asociados a la conducta sexual entre los usuarios de drogas admitidos en centros de asistencia y tratamiento de todo el Estado. Este objetivo general se hace operativo en los siguientes objetivos específicos para los TSMSs: a) ampliar los conocimientos sobre las vías de transmisión sexual del VIH y otras enfermedades infecto-contagiosas y sobre la prevención relacionada con las conductas sexuales; b) aumentar el autocontrol y planificación de las relaciones íntimas, y las habilidades para la negociación y resolución de problemas, y c) enseñar y entrenar el uso adecuado del preservativo y adecuar el balance costes-beneficios de cara a aumentar su utilización con la consiguiente disminución de las conductas sexuales de riesgo.

\subsection{Plan de Evaluación del Programa de Interven- ción con UDls}

\subsubsection{Objetivos de la evaluación}

La muestra está compuesta por los 25 TSMS para UDIs realizados por profesionales formados en nuestro programa de redes, cuyos conductores aceptaron entrar en un riguroso protocolo de evaluación. En estos han participado 387 UDIs en tratamiento en centros de atención a drogodependencias y fueron realizados durante el periodo enero-agosto del año 2001.

La evaluación de los talleres se centra en dos ámbitos: a) evaluación de los resultados e impacto de los talleres (eficacia), que evalúa tanto la evolución de las conductas sexuales de riesgo, como la modificación en las variables mediadoras que explican el cambio estas conductas (conocimientos, actitudes, expectativas); y b) evaluación de los procesos, cuyo objetivo es la valoración de la satisfacción de los usuarios de los talleres acerca de los diferentes elementos estructurales, impacto y satisfacción global con éstos.

Nuestro propósito es evaluar los resultados y procesos de los TSMS comparando los usuarios de éstos con UDIs en tratamiento que no han participado en los mismos.

\subsubsection{Hipótesis a contrastar en la evaluación de los Talleres}

Hipótesis principal: Los usuarios de los Talleres experimentarán cambios favorables tras la intervención (post-test) en relación con la línea base (pre-test), tanto en las conductas sexuales de riesgo como en las variables mediadoras de estas conductas, y esos cambios positivos tenderán a mantenerse tras el seguimiento. Por el contrario, estos cambios no serán experimentados por los sujetos que componen los grupos de comparación.

Hipótesis segunda: Los usuarios de los talleres no serán diferentes de los sujetos de comparación en las conductas de riesgo realizadas ni en las variables mediadoras evaluadas en el pre-test. Si existen diferencias en el post-test o seguimiento en estas variables, tales diferencias serán favorables a los usuarios de los talleres.

Hipótesis tercera: Los usuarios de los talleres mostrarán un nivel de satisfacción alto en relación con los diferentes elementos de los TSMSs en que han participado.

\subsubsection{Diseño de la evaluación}

El diseño de la evaluación es de tipo cuasi-experimental $2 \times 3$, con medidas repetidas y un grupo de control no equivalente (grupo de comparación). Los sujetos fueron asignados de forma no aleatoria a una de las dos condiciones (usuarios de los Talleres o grupo de comparación), teniendo en cuenta criterios de conveniencia clínica.

Los momentos de medida fueron tres:

1) Evaluación basal (pre-test), durante el primer día de implementación de los talleres.

2) Evaluación postest, inmediatamente tras la finalización de los talleres. 
Evaluación de seguimiento, un mes después de la finalización de los talleres, para evitar, en lo posible, la mortandad experimental característica de este tipo de estudios.

\subsubsection{Variables, instrumentos de medida y análisis estadísticos utilizados}

El instrumento utilizado para la recogida de información fue un cuestionario estructurado con preguntas cerradas agrupadas en las siguientes áreas: a) datos sociodemográficos (12 ítems); b) conocimientos sobre la transmisión y las medidas de protección frente al VIH/SIDA (30 ítems); c) conocimientos sobre el uso adecuado del preservativo (10 ítems); d) percepción de las ventajas y desventajas del preservativo (31 ítems) y expectativas de resultados esperados del uso del preservativo en las relaciones coitales (4 ítems), e) frecuencia del uso del preservativo en las relaciones coitales con pareja habitual y ocasional, f) porcentaje de relaciones coitales protegidas y problemas experimentados con el preservativo (5 ítems) y g) satisfacción de los usuarios de los TSMSs (10 ítems). Todos los instrumentos utilizados para la recogida de los datos expuestos en el presente informe han demostrado adecuadas consistencia interna y fiabilidad test-retest en su aplicación a los usuarios de los TSMS (Insúa, 2002).

Hemos utilizado los siguientes análisis estadísticos, realizados con el paquete estadístico SPSS, v.10.1.: a) análisis descriptivos (frecuencias y porcentajes, estimadores clásicos de localización y de escala media, desviación típica, etc.-); y b) análisis comparativos (tablas de contingencia y estadísticos asociados; comparación de medias basadas en la prueba t para muestras relacionadas -en la comparación intra-grupo a lo largo del estudio- y para muestras independientes -comparación inter-grupos en cada uno de los momentos de medida-; análisis de covarianza; pruebas no paramétricas para la comparación intra-grupo -la prueba de McNemar- y análisis de varianza de medidas repetidas). Se utilizaron también análisis log-lineales para detectar la evolución diferencial de ambos grupos en variables categóricas.

\subsubsection{Composición de la muestra.}

Como ya se ha señalado la muestra estaba formada por 387 sujetos, 226 hombres $(68,7 \%)$ y 121 mujeres $(31,3 \%)$, procedentes de programas de tratamiento de 13 Comunidades Autónomas (Aragón, Asturias, Baleares, Cantabria, Castilla-La Mancha, Castilla y León, Ceuta, C. Valenciana, Extremadura con la participación adicional de una ONG: UNAD-, Galicia, Madrid, Murcia y País Vasco); casi todos ellos de nacionalidad española (99\%). La media de edad es de 32,4 años (d.e. de 5,9 años). Algo más de la mitad están solteros $(58,4 \%)$, alrededor de la cuarta parte están casados o con pareja estable $(28,1 \%)$ y poco menos de la mitad tienen hijos (43,1\%). La mayoría de ellos tienen estudios equivalentes a la EGB o inferiores $(65,1 \%)$ y la mayoría de ellos están sin empleo o en situación de incapacidad laboral (54,6\% y 10,3\% respectivamente). La mitad han estado en prisión $(50,9 \%)$, la gran mayoría se han inyectado drogas alguna vez $(86,6 \%)$ y algo menos de la tercera parte reconocen ser seropositivos al VIH (31,9\%). Aproximadamente la mitad eran usuarios de los Talleres ( $\mathrm{N}=$ 193; 49,9\%) y componían por tanto el grupo de intervención, mientras que los demás formaban parte de los grupos de comparación ( $N=194 ; 50,1 \%$ ).

Las variables mediadoras utilizadas como medidas de resultados de los TSMSs son: a) conocimientos sobre el uso adecuado del preservativo, b) conocimientos sobre las situaciones de riesgo para la transmisión del VIH y para la protección frente a ésta; c) percepción de las ventajas e inconvenientes del preservativo, y expectativas de resultados de usar el preservativo.

Los indicadores conductuales utilizados como medidas de resultados de los TSMSs en el presente trabajo son: a) utilización siempre del preservativo con la pareja habitual; b) utilización siempre del preservativo con la pareja ocasional; c) porcentaje de relaciones coitales con preservativo; d) Problemas experimentados para convencer a la pareja para usar el preservativo; e) problemas experimentados con el uso del preservativo.

\section{RESULTADOS}

\subsection{Evaluación de resultados de los TSMS.}

\subsubsection{Conocimientos sobre el uso adecuado del preservativo}

Ambos grupos mostraban en el pre-test un nivel de conocimientos muy similar y notablemente elevado acerca de lo que constituye un uso adecuado del preservativo, respondiendo acertadamente a 8 de las 10 cuestiones (el 80\%).

En el post-test el nivel de conocimientos sobre el uso del preservativo es significativamente superior entre los usuarios de los TSMSs que entre el grupo de comparación, según la prueba T para la comparación de medias ( $t=4,9 ; 370$ g.l.; $p<0,000$ ). Lo mismo ocurre en el seguimiento ( $t=3,44 ; 322$ g.l.; $p<$ $0,001)$. Tanto en el post-test como en el seguimiento los usuarios de los TSMS alcanzan una puntuación equivalente a responder acertadamente a alrededor de 9 de las 10 cuestiones sobre los riesgos de la inyección (el 90\% de las cuestiones), mientras que el 
grupo de comparación responde acertadamente a algo más del $80 \%$ de las mismas.

El análisis univariante de la varianza, tomando como covariante las puntuaciones en el pre-test, demuestran que los usuarios de los TSMSs incrementan sus conocimientos sobre el uso adecuado del preservativo significativamente más que el grupo de comparación a lo largo del estudio en comparación con el pre-test. Esto es así tanto en el post-test ( $F=25,7 ; 1$ g.l.; $p<0,000)$ como en el seguimiento ( $F=10,2 ; 1$ g.l.; $p<0,002$ ). Los análisis de la varianza de medidas repetidas también demuestran que la evolución en el post-test en relación con el pre-test es más favorable entre los usuarios de los TSMS que entre los sujetos de comparación ( $F=10,2 ; 1$ g.l.; $p=0,002$ ) y se observa una tendencia al mismo efecto en el seguimiento en relación con el pre-test ( $F=3,1 ; 1 \mathrm{~g} . \mathrm{l}$; $p=0,08$ ). Estos datos proporcionan apoyo a la eficacia de los TSMS para aumentar los conocimientos acerca de lo que constituye un uso adecuado del preservativo (ver gráfico 1).

\subsubsection{Conocimientos sobre el SIDA}

El nivel de conocimientos sobre el SIDA era muy similar en los usuarios de los TSMSs y entre los sujetos del grupo de comparación en el pre-test, y pasó a ser superior entre los primeros frente a los segundos en el post-test y en el seguimiento $(t=4 ; 370$ g.l.; $p=$ 0,000 y $t=2,7 ; 355$ g.l.; $p=0,007$ respectivamente).

Los análisis univariados de la varianza con las medidas del pre-test como covariante demuestran que los usuarios de los TSMSs aumentan sus conocimientos sobre el SIDA tras los talleres significativamente más que los sujetos de comparación ( $F=22,4$; 1 g.l.; $p<$ $0,000)$. Lo mismo ocurre con los cambios tras el seguimiento en relación con el pretest $(F=8,1$; 1 g.l.; $p<$ $0,005)$. Los análisis de la varianza de medidas repetidas también demuestran que la evolución en el post-test en relación con el pre-test es más favorable entre los usuarios de los TSMS que entre los sujetos de comparación ( $F=15,7 ; 1$ g.l.; $p=0,000)$ y lo mismo ocurre en el seguimiento en relación con el pretest ( $F=5,3 ; 1$ g.l.; $p$ $=0,02$ ), proporcionando apoyo a la eficacia de los TSMS para aumentar los conocimientos sobre el SIDA entre sus usuarios. (Ver gráfico 2).

\subsubsection{Percepción de las ventajas e inconvenientes del preservativo}

Los usuarios de los TSMSs tenían antes de participar en los Talleres una percepción de las ventajas derivadas del uso del preservativo muy similar a los sujetos de comparación. Sin embargo, tras los talleres, hay un incremento significativo de la percepción de estas ven-

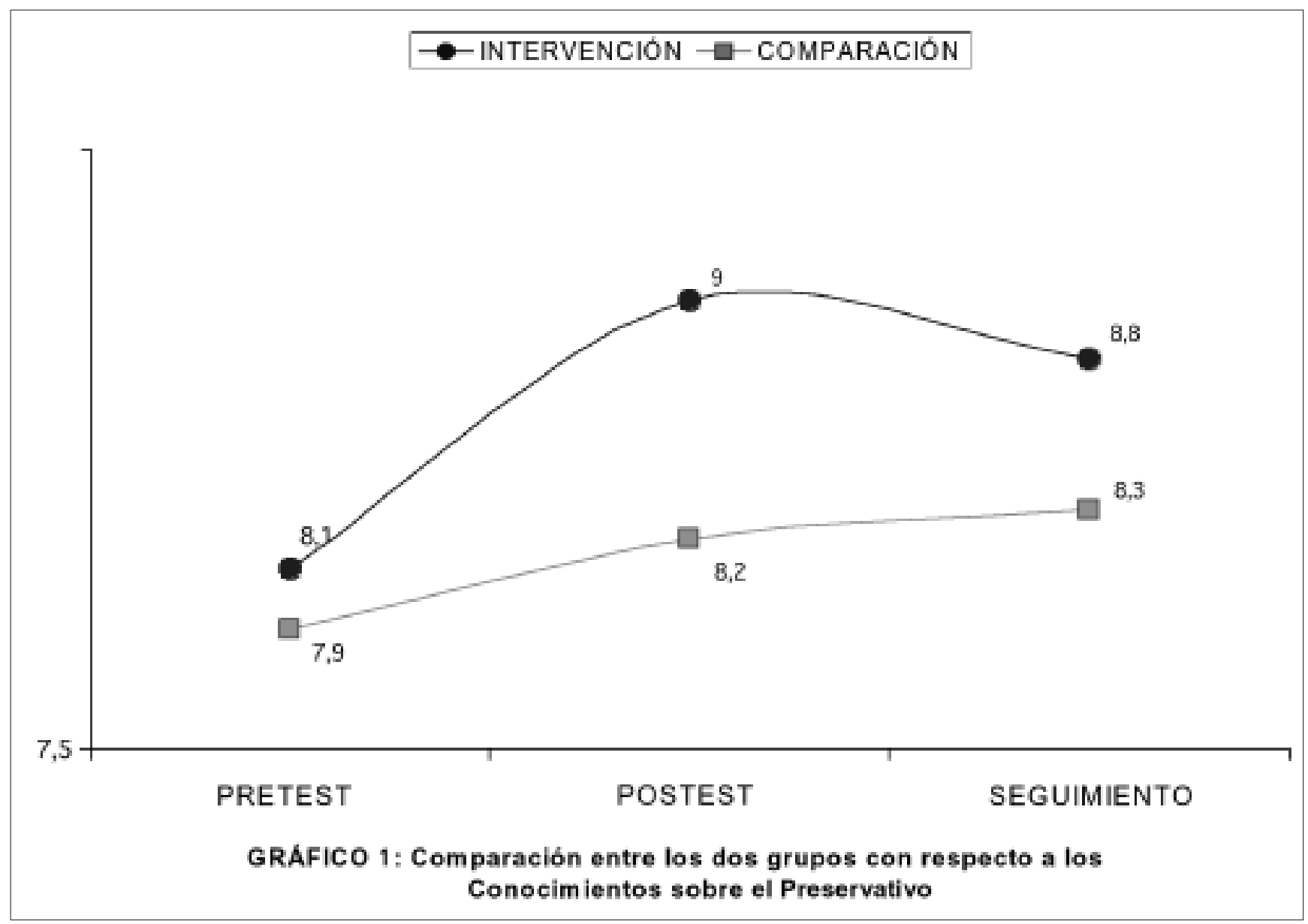




\section{INTERVENCIÓN - -COMPARACIÓN}

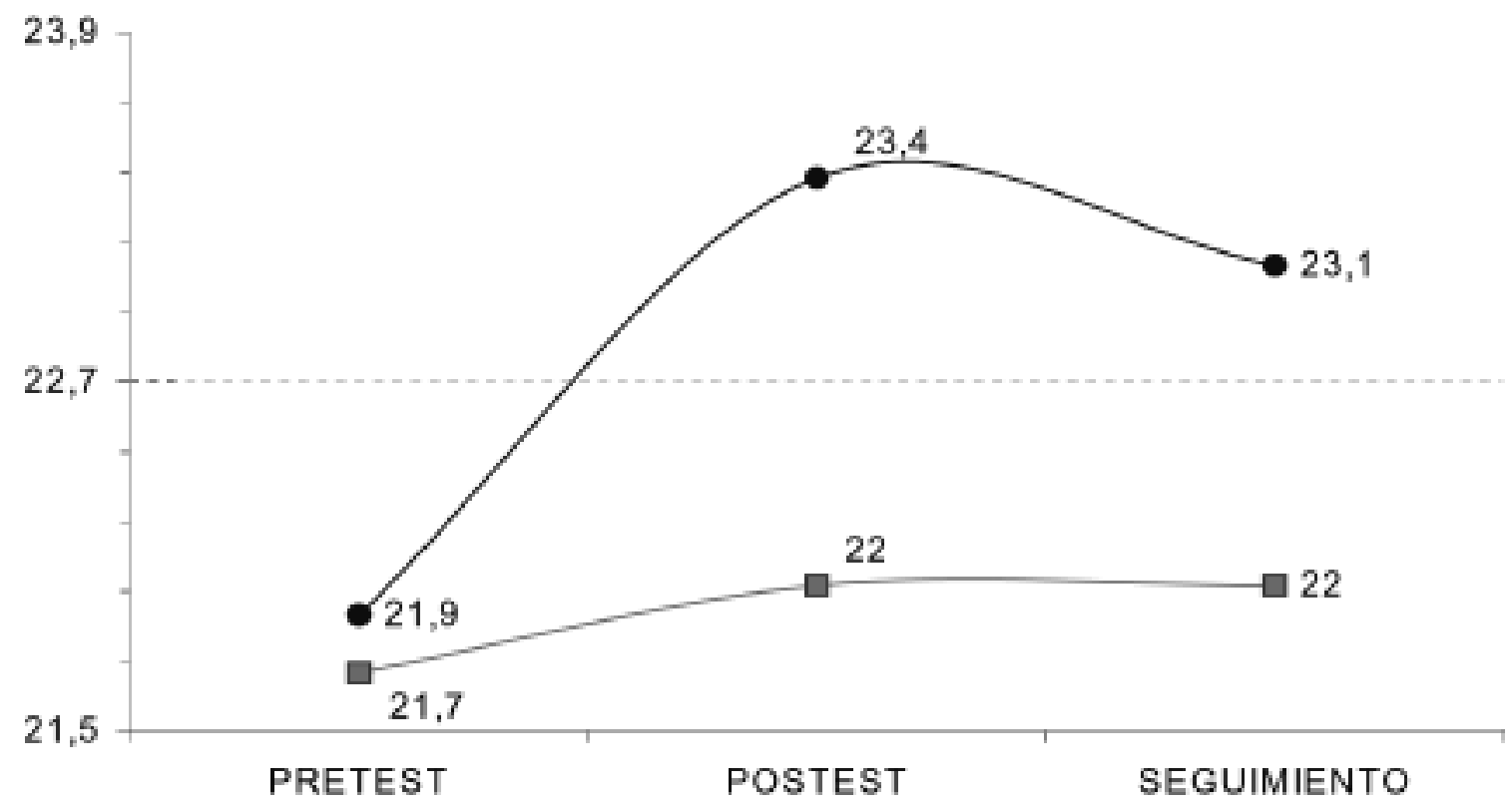

\section{GRÁFICO 2: Comparación entre los dos grupos con respecto a los Conocimientos sobre VIH / SIDA}

tajas entre los sujetos del grupo de intervención, tanto en el post-test como en el seguimiento a 1 mes $(t=2,3$; 370 g.l.; $p=0,02$ y $t=2 ; 355$ g.l.; $p=0,047$ respectivamente). Los análisis de covarianza con las medidas de pre-test como covariante demuestran que los usuarios de los TSMS aumentan significativamente más su percepción de las ventajas del preservativo en comparación con el pre-test que los sujetos de comparación, y esto ocurre tanto en el post-test como en el seguimiento ( $F$ $=5,8$; 1 g.l.; $p=0,02$ y $F=3,8 ; 1$ g.l.; $p=0,05$ respectivamente). En conclusión: estos resultados proporcionan apoyo a la eficacia de los TSMS para aumentar la percepción de las ventajas del preservativo (ver gráfico 3)

Con respecto a la percepción sobre las desventajas del preservativo, no existen diferencias significativas entre ambos grupos en el pre-test $(t=1,16$; 385 g.l.; $p$ $=0,25)$; mientras que tras los TSMSs, los usuarios de los talleres disminuían la percepción de estas desventajas significativamente más que los sujetos de comparación ( $F=5,8 ; 1$ g.l.; $p=0,017)$. Como consecuencia, al finalizar los TSMSs la percepción de las desventajas del preservativo era significativamente menor entre los usuarios de estos talleres que entre los sujetos de comparación ( $t=2,6$; 370 g.l.; $p=0,009$ ).

Durante el seguimiento, no se mantiene la tendencia observada entre los usuarios de los Talleres. Estos resultados apoyan la eficacia de los TSMSs para disminuir la percepción de las desventajas del preservativo, aunque esta disminución tiende a no mantenerse durante el seguimiento si se compara con la evolución de los sujetos que no participaron en estos Talleres (ver gráfico 4).

La escala de Ventajas e Inconvenientes del Preservativo permite obtener una puntuación relativa al balance decisional sobre el uso del preservativo (Balance = ventajas - desventajas). Los análisis de varianza de medidas repetidas demuestran también que los usuarios de los TSMS evolucionan más favorablemente que los sujetos de comparación entre el pre-test y el post-test ( $F=5,4 ; 1$ g.l.; $p=0,021)$ en esta puntuación, aunque esas diferencias no se mantienen en el seguimiento. En resumen, estos resultados aportan evidencia favorable a la eficacia de estos Talleres para positivar el balance decisional relativo al uso del preservativo, si bien el impacto desciende con el tiempo.

\subsubsection{Expectativas de resultados relacionados con el uso del preservativo.}

Las expectativas de resultados e impacto del uso del preservativo sobre las relaciones sexuales fueron evaluadas con dos dimensiones positivas (seguras y lúdicas) y con dos dimensiones negativas (limitadas e incómodas). Los encuestados respondían sobre una escala que mostraba su acuerdo o desacuerdo con tales calificativos. La Tabla $N^{\circ} 1$ muestra las frecuen- 

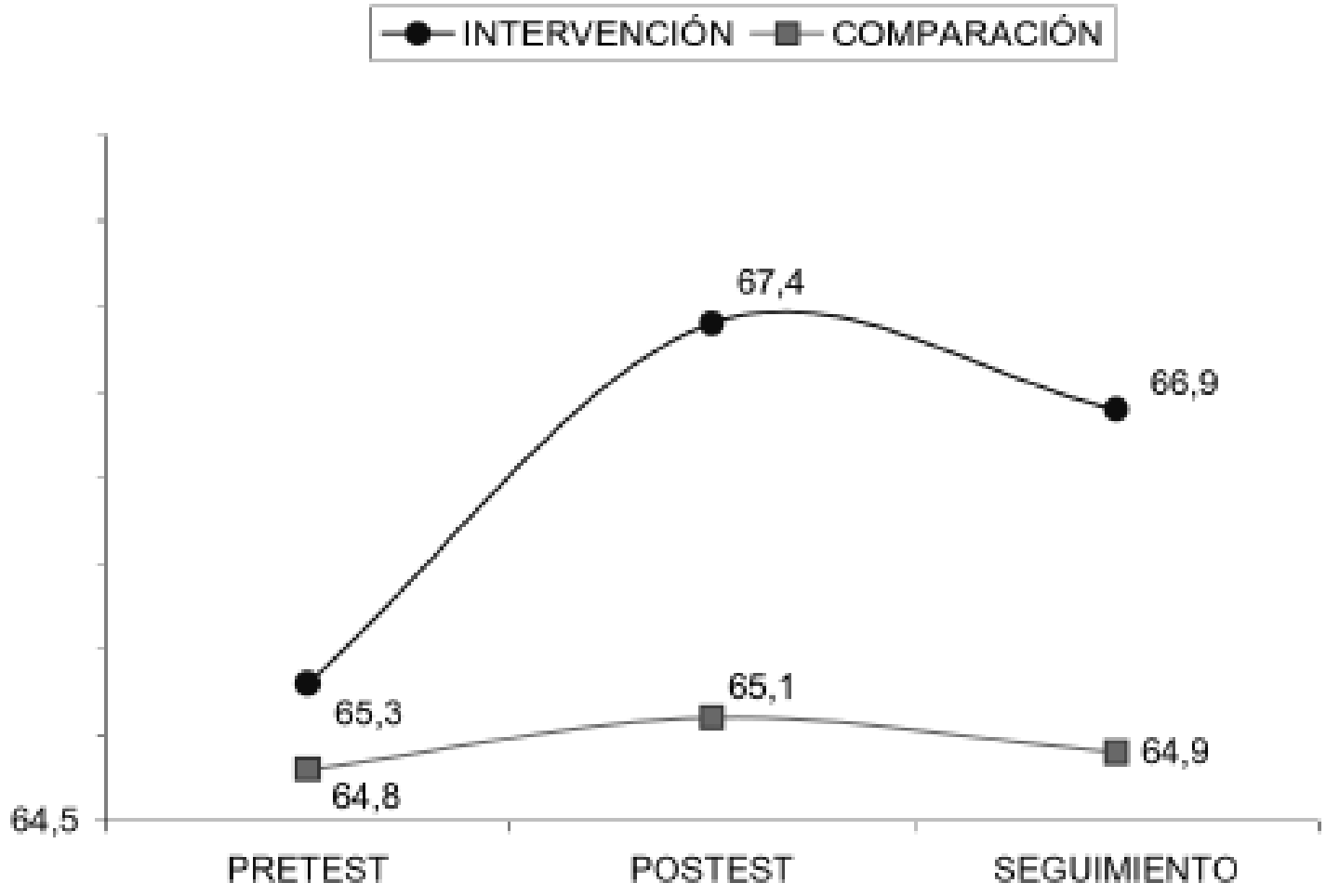

GRÁFICO 3: Comparación entre los dos grupos con respecto a los Beneficios del Preservativo

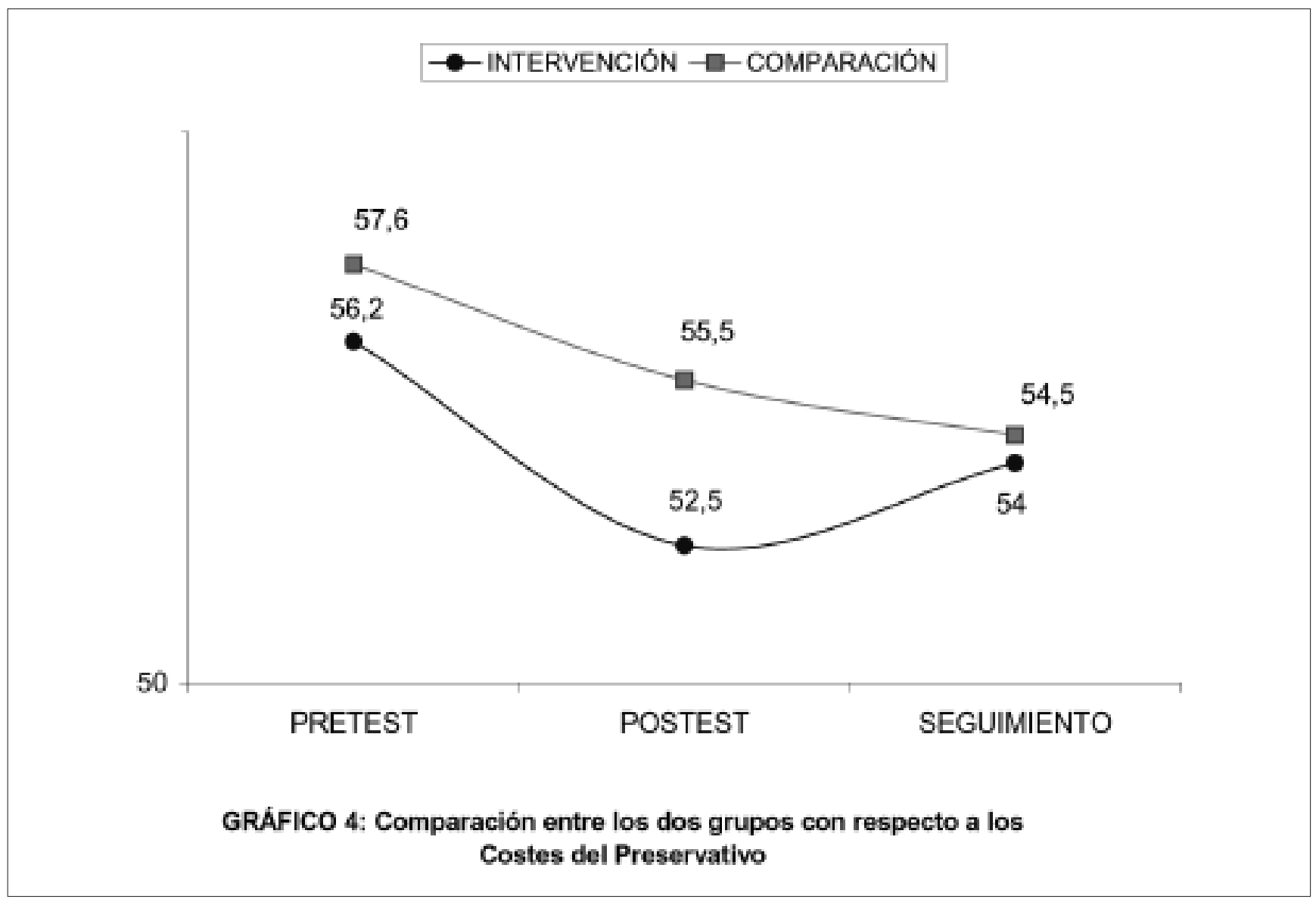


cias y porcentajes de sujetos que se mostraron de acuerdo con esos cuatro calificativos aplicados a lo que esperan de sus relaciones sexuales con preservativo y los estadísticos de contraste (bilateral) para la comparación entre ambos grupos en cada evaluación, La Tabla No 2 muestra los estadísticos de contraste (bilateral) sobre la evolución de ambos grupos a lo largo del estudio según la prueba de McNemar.

Los resultados mostrados en las Tablas $N^{\circ} 1$ y N $N^{\circ}$, acerca de las expectativas sobre los resultados e impacto del preservativo en las relaciones sexuales indican que: a) los usuarios de los TSMSs tienden a aumentar su percepción de seguridad en las relaciones sexuales protegidas tras los Talleres, aunque no hay diferencias significativas entre el seguimiento y el pretest; b) solamente la mitad o menos de los encuestados consideran que las relaciones sexuales con preservativo serán lúdicas y agradables. Ambos grupos mejoran esas expectativas significativamente en el post-test y en el seguimiento en relación con el pretest, y esa mejoría es mayor entre los usuarios de los TSMSs; c) alrededor de la mitad de los encuestados consideran que el uso del preservativo ocasionará que sus relaciones sexuales sean limitadas, aunque los usuarios de los TSMSs disminuyen significativamente esas expectativas negativas durante los talleres; d) la mitad o más de los encuestados espera que el uso del preservativo ocasionará que sus relaciones sexuales sean incómodas. Los usuarios de los TSMSs disminu- yen significativamente esas expectativas negativas durante los talleres, hasta el punto en que en el posttest esas expectativas son significativamente menos frecuentes que en el grupo de comparación. Al finalizar el estudio aún se mantienen las diferencias significativas entre los usuarios de los TSMSs y los sujetos de comparación, indicando que los últimos mantienen mayores expectativas de que el uso del preservativo ocasione que sus relaciones sexuales sean incómodas. Los análisis log-lineales indican que los usuarios de los TSMSs reducen, entre el pre-test y el seguimiento, significativamente más que los sujetos de comparación dos expectativas negativas sobre el uso del preservativo: las expectativas de que sus relaciones coitales serán limitadas e incómodas por utilizar el preservativo (Chi cuadrado $=4,5 ; p=0,03$ y Chi cuadrado $=8,6 ; p=0,003$ respectivamente).

En conclusión, la participación en los TSMSs va asociada a una mejora en las expectativas de los resultados asociados al uso del preservativo, que se concreta en un aumento de las expectativas positivas y una disminución de las expectativas negativas de los resultados del uso del preservativo.

\subsubsection{Uso del preservativo con pareja habitual.}

En el pre-test, la conducta más frecuente en ambos grupos es no utilizar nunca el preservativo en sus relaciones con la pareja habitual (38,5\% y 50,3\%

Tabla $n^{\circ}$ 1. Expectativas acerca del impacto del preservativo en las relaciones coitales y estadísticos de contraste relativos a la comparación transversal entre grupos.

\begin{tabular}{|c|c|c|c|c|c|c|c|c|}
\hline \multirow[b]{2}{*}{ Ítem } & \multirow[b]{2}{*}{ Evaluación } & \multicolumn{2}{|c|}{ Experimental } & \multicolumn{2}{|c|}{ Comparación } & \multicolumn{3}{|c|}{ Contraste } \\
\hline & & $\mathbf{N}$ & $\%$ & $\mathbf{N}$ & $\%$ & Chi & g.l. & $P$ exacta \\
\hline \multirow[t]{3}{*}{ Relaciones seguras } & Pre-test & 173 & 92 & 172 & 95 & 1,4 & 1 & 0,29 \\
\hline & Post-test & 175 & 96,7 & 175 & 95,1 & 0,58 & 1 & 0,6 \\
\hline & Seguimiento & 168 & 93,9 & 159 & 93,5 & 0,02 & 1 & 1 \\
\hline \multirow[t]{3}{*}{ Relaciones lúdicas } & Pre-test & 57 & 33,3 & 51 & 30,7 & 0,26 & 1 & 0,64 \\
\hline & Post-test & 91 & 57,2 & 72 & 41,9 & 7,8 & 1 & 0,006 \\
\hline & Seguimiento & 85 & 53,1 & 63 & 38,9 & 6,6 & 1 & 0,014 \\
\hline \multirow[t]{3}{*}{ Relaciones limitadas } & Pre-test & 99 & 55,6 & 84 & 49,1 & 1,5 & 1 & 0,24 \\
\hline & Post-test & 62 & 37,6 & 78 & 45,6 & 2,23 & 1 & 0,15 \\
\hline & Seguimiento & 57 & 34,5 & 63 & 38,7 & 0,6 & 1 & 0,49 \\
\hline \multirow[t]{3}{*}{ Relaciones incómodas } & Pre-test & 116 & 64,4 & 118 & 65,9 & 0,09 & 1 & 0,83 \\
\hline & Post-test & 76 & 44,7 & 105 & 60,3 & 8,4 & 1 & 0,005 \\
\hline & Seguimiento & 70 & 41,9 & 89 & 53,6 & 4,6 & 1 & 0,037 \\
\hline
\end{tabular}


Tabla $\mathbf{n}^{\circ}$ 2. Evolución de las expectativas sobre el impacto del preservativo en las relaciones sexuales a lo largo del estudio: Comparación entre los estadísticos de ambos grupos según la prueba de McNemar.

\begin{tabular}{||l|l|c|c|c|c||}
\hline \multicolumn{2}{|c|}{ Ítem } & \multicolumn{2}{|c|}{ Experimental } & \multicolumn{2}{c|}{ Comparación } \\
\hline Relaciones seguras & \multicolumn{1}{|c|}{ Evaluación } & $\mathbf{N}$ & $\mathbf{P}$ & $\mathbf{N}$ & $\mathbf{P}$ \\
\cline { 2 - 7 } & Pre-test - Post-test & 177 & 0,092 & 173 & 1 \\
\cline { 2 - 7 } & Postest - Seguimiento & 172 & 0,39 & 168 & 0,75 \\
\cline { 2 - 7 } & Pretest - Seguimiento & 176 & 0,50 & 161 & 0,55 \\
\hline Relaciones lúdicas & Pre-test - Postest & 149 & 0,000 & 150 & 0,049 \\
\cline { 2 - 7 } & Postest - Seguimiento & 142 & 0,44 & 153 & 1 \\
\cline { 2 - 7 } & Pretest - Seguimiento & 146 & 0,000 & 148 & 0,027 \\
\hline Relaciones limitadas & Pre-test - Post-test & 156 & 0,000 & 153 & 0,66 \\
\cline { 2 - 7 } & Postest - Seguimiento & 150 & 0,39 & 153 & 0,19 \\
\cline { 2 - 7 } & Pretest - Seguimiento & 155 & 0,000 & 149 & 0,040 \\
\hline Relaciones incómodas & Pre-test - Post-test & 160 & 0,000 & 161 & 0,29 \\
\cline { 2 - 7 } & Postest - Seguimiento & 156 & 0,42 & 157 & 0,14 \\
\cline { 2 - 7 } & Pretest - Seguimiento & 159 & 0,000 & 154 & 0,001 \\
\hline \hline
\end{tabular}

en los usuarios de los TSMSs y grupos de comparación respectivamente). En el post-test, aunque aumenta notablemente en ambos grupos en relación con el pre-test el uso del preservativo siempre con la pareja habitual $(36,4 \%$ y $30,9 \%$ en los usuarios de los TSMS y grupos de comparación respectivamente), la conducta más frecuente en ambos grupos continúa siendo no utilizar nunca el preservativo en estas relaciones,. En el seguimiento, la conducta más frecuente en el grupo de usuarios de los TSMSs es utilizar siempre el preservativo en sus relaciones con la pareja habitual (36,9\%), mientras que la mayoría de los sujetos de comparación siguen sin utilizar nunca el preservativo en estas relaciones $(54,7 \%)$. Los grupos de usuarios de los TSMSs no se distinguen significativamente de los sujetos de comparación en la frecuencia de uso del preservativo en las relaciones coitales con la pareja habitual durante el pre-test y el post-test. Sin embargo, la frecuencia de uso del preservativo durante el seguimiento es significativamente mayor en el grupo experimental que en el grupo de comparación (Chi $=10,2 ; 3$ g.l.; p bilateral estimada mediante el procedimiento de Monte Carlo $=0,017$ ) .
Los estadísticos de contraste basados en la prueba de McNemar nos permiten detectar que en el grupo experimental aumenta significativamente la proporción de sujetos que utilizan siempre el preservativo con su pareja habitual en el post-test con relación al pre-test ( $p=0,021$ para 96 sujetos con este tipo de relación en pre-test y post-test), y que estas diferencias positivas y significativas se mantienen durante el seguimiento ( $p=0,021$ para 105 sujetos con este tipo de relación en pre-test y seguimiento).

Los TSMSs han mostrado utilidad para aumentar significativamente la probabilidad de que los sujetos utilicen el preservativo siempre en sus relaciones coitales con la pareja habitual, y esos aumentos se mantienen tras un mes de seguimiento (ver Gráfico № 5 ).

\subsubsection{Uso del preservativo con pareja ocasional}

Con respecto a las relaciones con pareja ocasional, en el pre-test, la conducta más frecuente en ambos grupos es utilizar siempre el preservativo en sus relaciones con ésta, a diferencia de lo que ocurría en las 


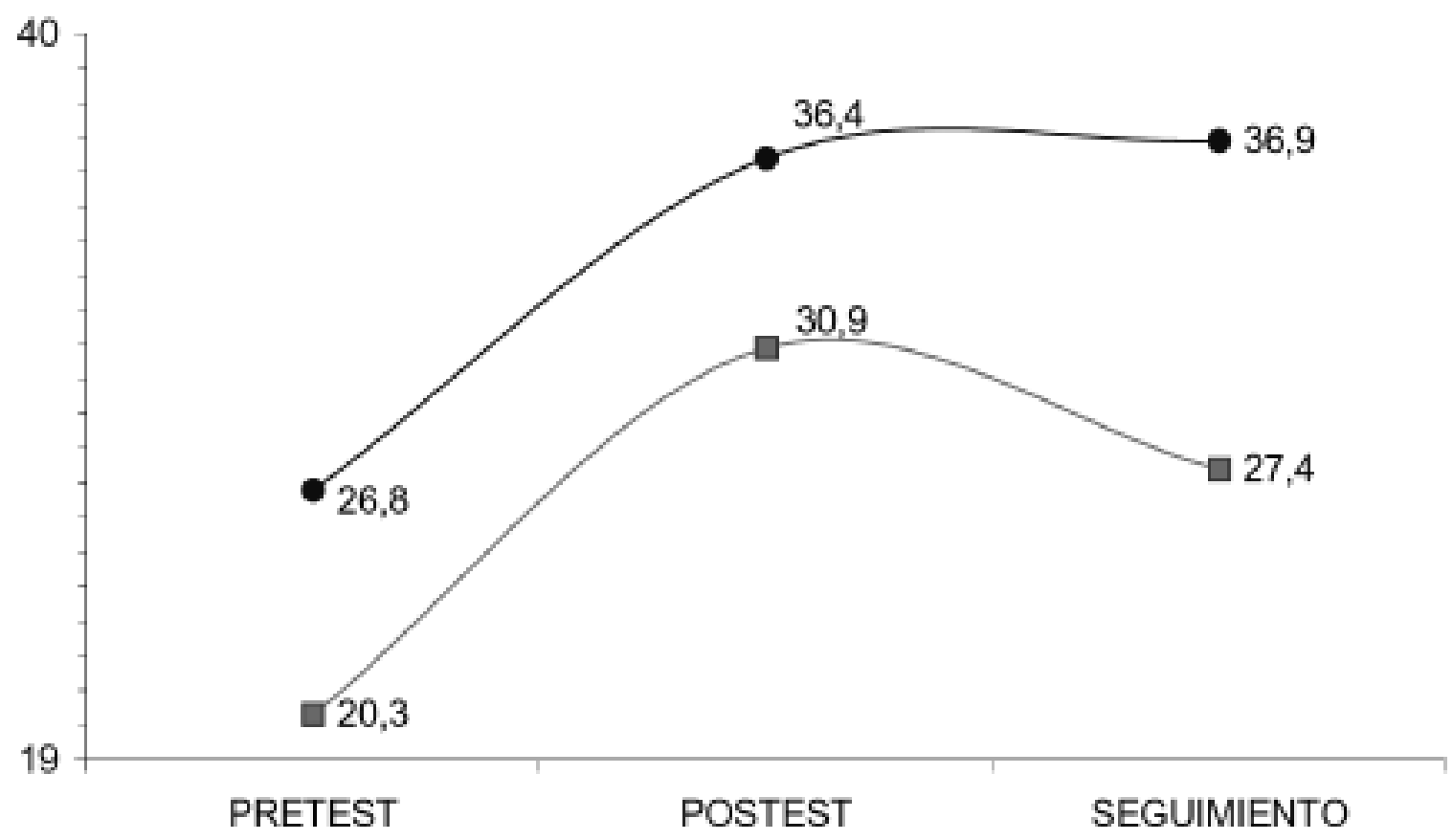

\section{GRÁFICO 5: Comparación entre los dos grupos en el uso siempre de preservativo con pareja habitual $(\%)$}

relaciones con pareja habitual $448,3 \%$ y $49,5 \%$ en los usuarios de los TSMSs y grupos de comparación respectivamente). En el post-test, la conducta más frecuente en ambos grupos continúa siendo utilizar siempre el preservativo en estas relaciones coitales con pareja ocasional, aumentando en ambos grupos en relación con el pre-test la frecuencia de esta conducta de protección, aunque más notablemente entre los usuarios de los TSMSs $(70,4 \%$ y $59,1 \%$ en los usuarios de los TSMSs y grupos de comparación respectivamente). En el seguimiento, los sujetos de comparación se mantienen con las mismas probabilidades que en el post-test de usar el preservativo en estas relaciones $(57,1 \%)$, mientras que los usuarios de los TSMSs retornan a niveles ligeramente inferiores a los del pretest en esta conducta de protección $(36,4 \%)$. Sin embargo, los grupos de usuarios de los TSMSs no se distinguen a nivel estadístico de los sujetos de comparación en la frecuencia de uso del preservativo en las relaciones coitales con la pareja ocasional durante el pre-test, en el post-test ni en el seguimiento.

Los estadísticos de contraste basados en la prueba de McNemar con el nivel de significación exacto nos permiten detectar que en el grupo experimental aumenta significativamente la proporción de sujetos que utilizan siempre el preservativo con su pareja ocasional en el post-test con relación al pre-test $(p=$
0,031 para 22 sujetos con este tipo de relación en pretest y post-test), y que estas diferencias positivas y significativas no se mantienen durante el seguimiento ( $p=0,29$ para 28 sujetos con este tipo de relación en pretest y seguimiento) (ver Gráfico N6).

\subsubsection{Porcentaje de relaciones coitales protegidas.}

Antes del desarrollo de los talleres, el porcentaje de relaciones coitales protegidas (durante los últimos 3 meses) era levemente inferior al 50\% en ambos grupos, sin diferencias significativas entre ellos. En el post-test, el porcentaje de relaciones coitales protegidas (durante el periodo de duración de los talleres) alcanzó casi el 60\% en el grupo experimental global y superó ligeramente el $50 \%$ en el grupo de comparación, sin que esas diferencias fuesen estadísticamente significativas. Al finalizar el estudio, el porcentaje de relaciones coitales protegidas se mantenía sobre el $60 \%$ en el grupo experimental y nuevamente bajaba hasta niveles semejantes a la línea base en el grupo de comparación. En este caso, las diferencias de medias son estadísticamente significativas, a favor del grupo de intervención ( $t=2,36 ; 204,8$ g.l.; $p=0,019$ ).

Tanto los usuarios de los TSMSs como los sujetos de comparación aumentan significativamente el por- 


\section{-INTERVENCIÓN $\rightarrow$-COMPARACIÓN}

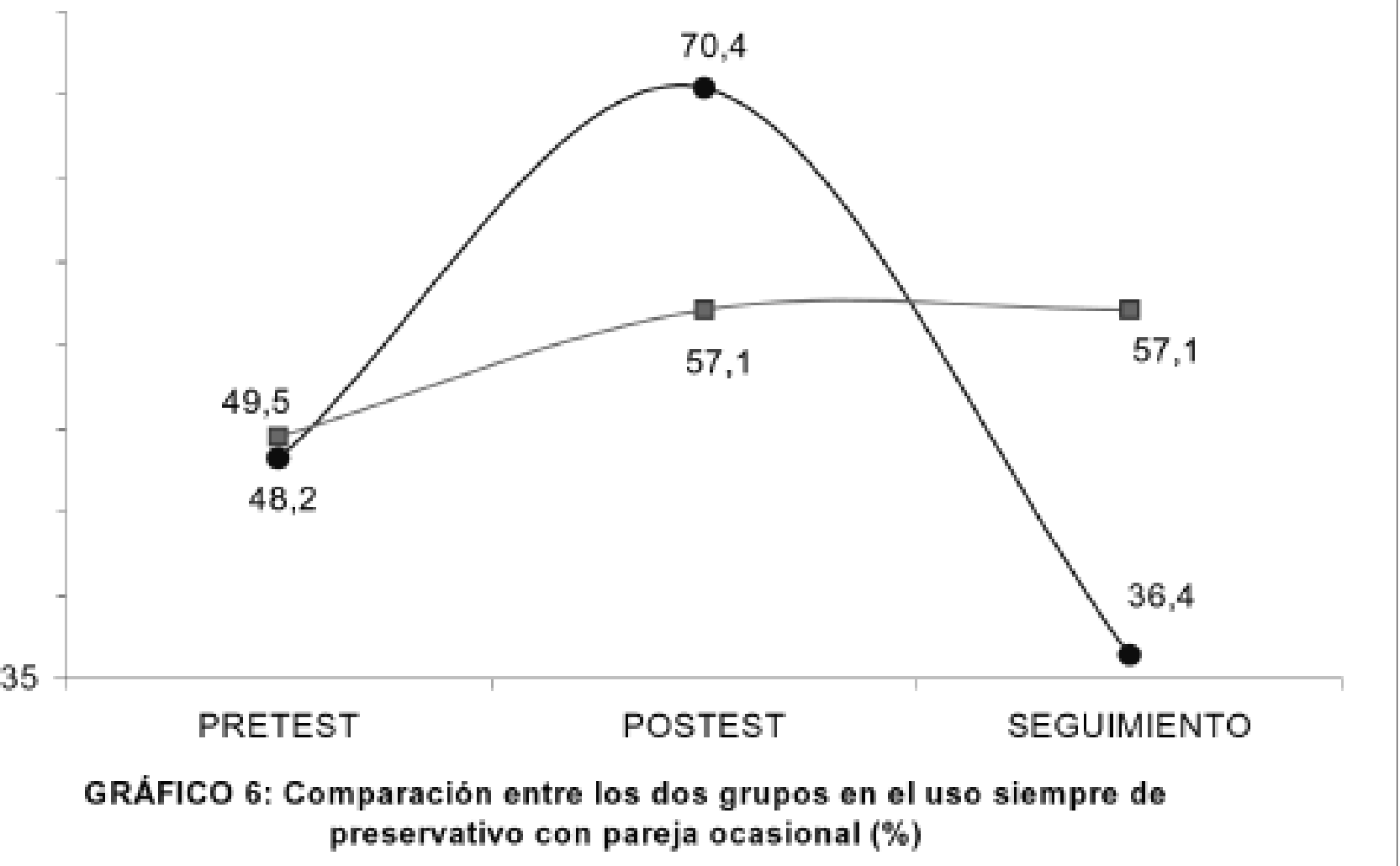

centaje de relaciones coitales protegidas durante el post-test ( $t=2,14 ; 82$ g.l.; $p=0,035$ para el grupo de usuarios de los TSMSs) y el seguimiento $(t=2,38 ; 93$ g.l.; $p=0,019$ para el grupo de usuarios de los TSMSs) en relación con el pret-est. El análisis univariante de la varianza en el post-test, utilizando como covariante las medidas del pre-test nos indica que la evolución de esta variable durante el estudio no muestra diferencias significativas entre ambos grupos. Esta variable muestra una estabilidad intra-sujetos considerable y muy significativa estadísticamente a lo largo de todo el estudio: La correlación entre las medidas en el pretest y en el post-test alcanzó un valor de 0,80 en el grupo experimental y en el grupo de comparación y los coeficientes de correlación entre el pre-test y el seguimiento fueron de 0,73 en el grupo experimental y de 0,70 en el grupo de comparación.

En conclusión, el porcentaje de relaciones coitales protegidas se sitúa entre el $40 \%$ y el $60 \%$ a lo largo del estudio en ambos grupos. Tanto los usuarios de los TSMSs como los sujetos de comparación aumentan significativamente ese porcentaje tras los Talleres y en el seguimiento en comparación con el pre-test (ver Gráfico № 7), por lo que esos efectos positivos no pueden ser atribuidos inequívocamente al impacto de los TSMSs. No obstante, la evolución a lo largo de estudio en esta conducta de protección es más favo- rable entre los usuarios de los TSMSs que entre los sujetos de comparación, hasta el punto que aquellos incrementan este porcentaje de relaciones coitales protegidas el doble que estos últimos en el seguimiento en relación con el pre-test (21\% vs. 10,5\%).

\section{1.8. Problemas experimentados con el uso del preservativo.}

Los sujetos entrevistados informaron sobre sus problemas a la hora de convencer a la pareja de la necesidad de usar preservativo en las relaciones sexuales y sobre problemas experimentados con el uso del preservativo. La Tabla $N^{\circ} 3$ muestra las frecuencias y porcentajes de sujetos en cada uno de los dos grupos que experimentaron al menos alguna vez problemas con el uso del preservativo entre aquellos sujetos que mantuvieron relaciones durante cada uno de los tres periodos de medida. En ninguno de los tres momentos de medida se observaron diferencias significativas entre grupos en la frecuencia de estos dos tipos de problemas con el preservativo, según los estadísticos de contraste aplicados a las tablas de contingencia.

Con respecto a los problemas para convencer a la pareja acerca de la necesidad de usar el preservativo, éstos son relativamente poco frecuentes a lo largo del estudio: aproximadamente uno de cada tres sujetos 


\section{-INTERVENCIÓN $\rightarrow$-COMPARACIÓN}

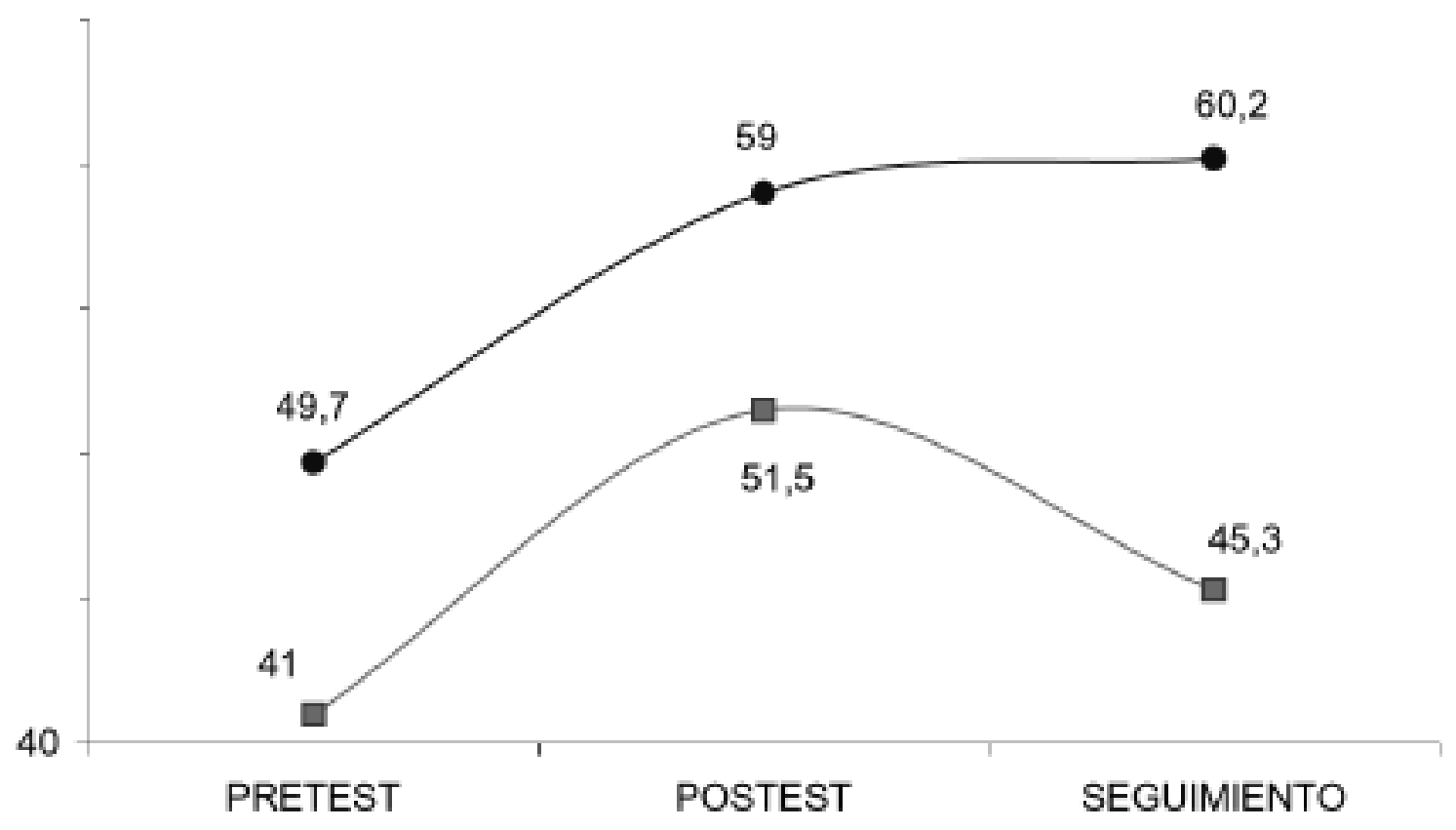

\section{GRÁFICO 7: Comparación entre los dos grupos con respecto al Porcentaje de relaciones coitales protegidas}

informan de algún problema de este tipo en el pre-test, y alrededor de uno de cada cinco en el post-test y el seguimiento. En ninguno de estos tres momentos de medida se observan diferencias significativas entre los usuarios de los TSMSs y los sujetos de comparación.

En conclusión, aunque los problemas para usar el preservativo y para convencer de la necesidad de su uso en las relaciones sexuales se habían producido en un porcentaje menor del $50 \%$ de los sujetos en los últimos 3 meses y menor del $25 \%$ durante el mes y medio del estudio, demostrando un descenso significativo tras los TSMSs en comparación con el pre-test, no se puede afirmar que los TSMSs han reducido significativamente la frecuencia de estos problemas, ya que la disminución es la misma en ambos grupos.

\subsection{Evaluación de la Satisfacción de los usuarios con los TSMSs}

Cada uno de los 10 ítems del cuestionario de satisfacción se responde sobre una escala cuyos valores máximo y mínimo son 5 y 1 (mucho y nada satisfecho respectivamente).

La satisfacción del usuario está agrupada en tres dimensiones:
1. Satisfacción relativa a la adecuación del taller a las características del usuario: momento personal, interés suscitado, utilidad para el cambio en las conductas de riesgo y satisfacción global.

2. Satisfacción relativa a aspectos específicos del taller: adecuación del local, metodología de exposición de los temas, adecuación del grupo e interés de este tipo de Talleres para un futuro.

3. Satisfacción con los recursos humanos del taller: competencia y accesibilidad del personal responsable de los Talleres.

La Tabla $N^{\circ} 4$ resume las puntuaciones promedio relativas a la satisfacción de los usuarios de los TSMSs, tanto en el post-test como en el seguimiento.

Los resultados que pueden observarse en la tabla anterior indican que: a) el nivel de satisfacción de los TSMSs es alto en todos los aspectos de los Talleres; b) que ese alto nivel de satisfacción tiende a mantenerse o incluso a aumentar en el seguimiento; c) que los ítems con menor nivel de satisfacción son los relativos a la preparación personal de los propios usuarios para el Taller y la adecuación de los locales y d) que los ítems con un nivel de calificación promedio cercano al máximo son los relativos a la proyección futura de estos talleres y los atributos del personal responsable de los mismos. 
Tabla $\mathbf{n}^{\circ}$ 3. Problemas con el preservativo a lo largo del estudio.

\begin{tabular}{||l|l|c|c|c|c||}
\hline & & \multicolumn{2}{|c||}{ Experimental } & \multicolumn{2}{c|}{ Comparación } \\
\hline \multicolumn{1}{|c|}{ Ítem } & Evaluación & $\mathbf{N}$ & $\mathbf{\%}$ & $\mathbf{N}$ & $\%$ \\
\hline \multirow{2}{*}{$\begin{array}{l}\text { Problemas para convencer de la necesidad de usar el } \\
\text { preservativo }\end{array}$} & Pre-test & 71 & 37,8 & 71 & 38,4 \\
\cline { 2 - 7 } & Post-test & 24 & 18,1 & 30 & 23,1 \\
\cline { 2 - 7 } & Seguimiento & 32 & 22,5 & 21 & 16,1 \\
\hline \multirow{3}{*}{ Problemas con el manejo y uso del preservativo } & Pre-test & 76 & 40,2 & 77 & 41,1 \\
\cline { 2 - 7 } & Post-test & 12 & 9,5 & 5 & 4,1 \\
\cline { 2 - 7 } & Seguimiento & 9 & 6,4 & 7 & 5,5 \\
\hline
\end{tabular}

Tabla $n^{\circ}$ 4. Satisfacción promedia de los usuarios de los TSMS, en el postest y el seguimiento

\begin{tabular}{||l|c|c||}
\hline ITEM & Post-test & Seguimiento \\
\hline Momento personal & 3,6 & 3,8 \\
\hline Interés suscitado & 4,3 & 4,2 \\
\hline $\begin{array}{l}\text { Utilidad para cambiar las conductas de } \\
\text { riesgo }\end{array}$ & 4 & 4,2 \\
\hline Satisfacción general con el taller & 4,3 & 4,4 \\
\hline Adecuación del local & 3,8 & 3,8 \\
\hline Método para la explicación de los temas & 4,3 & 4,3 \\
\hline Adecuación de la composición del grupo & 4,2 & 4,1 \\
\hline Interés del Taller para un futuro & 4,6 & 4,6 \\
\hline Competencia y conocimientos del personal & 4,4 & 4,5 \\
\hline Accesibilidad del personal & 4,5 & 4,5 \\
\hline \hline
\end{tabular}

Concluyendo, los usuarios de los TSMSs valoran muy positivamente estos talleres, tanto a su finalización como un mes después de haber finalizado. Estos talleres podrían mejorarse incorporando una fase introductoria para preparar y motivar a los usuarios, y su generalización sería muy bien recibida por el colectivo de drogodependientes en tratamiento.

\section{DISCUSIÓNY CONCLUSIONES}

Existe la idea generalizada de que hoy en día la información sobre sexualidad y aspectos relacionados a la protección de las relaciones sexuales es relativamente amplia. En nuestro estudio hemos observado que el nivel de conocimientos promedio sobre el uso adecuado del preservativo es muy alto y que el conocimiento sobre las formas de transmisión del $\mathrm{VIH} / \mathrm{SIDA}$ y las conductas de protección frente a esta transmisión es más moderada, resultados que permiten afirmar que la mayoría de los sujetos drogodependientes en tratamiento que participaron en la evaluación de los TSMSs disponen de información correcta sobre el uso adecuado del preservativo y sobre la transmisión del VIH/SIDA, junto con algunas informaciones incorrectas que son resistentes a su modificación. Indirectamente, nuestro estudio también avala la hipótesis de que disponer de información adecuada sobre el preservativo y sobre la transmisión del VIH/SIDA no se corresponde con la adopción de medidas preventivas, como se demuestra con las bajas tasas de protección en las relaciones coitales. Nuestros resultados indican claramente que los usuarios de los TSMSs aumentan significativamente sus 
conocimientos sobre el uso del preservativo y sobre la transmisión del VIH/SIDA durante los Talleres en comparación con la línea base, que lo hacen en una medida significativamente mayor que los sujetos que no participaron en dichos Talleres y que esos aumentos en los conocimientos tienden a mantenerse en el seguimiento. Aunque no se pueda asegurar que ese aumento en los conocimientos se traduzca en menores conductas sexuales de riesgo, es de esperar que ese aumento tenga al menos un efecto indirecto positivo hacia el cambio en la conducta riesgo.

Nuestros resultados demuestran también claramente que los usuarios de los TSMS mejoran durante los Talleres en sus actitudes relativas al balance costes-beneficios sobre las consecuencias del uso del preservativo y que ese aumento en la percepción de las ventajas se mantiene en el seguimiento entre los usuarios de los TSMSs, aunque no ocurre así con la disminución de las desventajas percibidas del preservativo. Sin embargo, las expectativas negativas sobre el impacto del preservativo en las relaciones sexuales disminuyen, y las expectativas positivas aumentan entre los usuarios de los TSMSs y los cambios son superiores a los observados en los sujetos del grupo de comparación. Algunos estudios indican que las actitudes y expectativas de resultados relacionadas con la relación de conductas sexuales preventivas predicen positivamente la realización de tales conductas (Corby, Schneider y Wolitski, 1996; Falck et al., 1997). Estas mejorías en las actitudes hacia el preservativo y en las expectativas sobre sus resultados pueden jugar un papel importante en el mantenimiento de las conductas de protección y pueden ser una de las causas de que las mejorías en las conductas observadas durante los TSMSs se mantengan en el seguimiento, en la medida en que es reconocida la relación entre actitudes, expectativas de resultado y realización de la conducta preventiva.

Una primera valoración de los resultados observados en cuanto al uso del preservativo en las relaciones coitales indica que los usuarios de los TSMSs alcanzan un incremento en estos indicadores de conductas de protección entre el pre-test y el post-test que supera el $10-20 \%$ de aumento informado en la literatura sobre la evaluación de intervenciones de naturaleza similar a los TSMSs (Páez et al, 1996). El incremento observado es del $37,7 \%$ en el uso del preservativo siempre con pareja habitual (pasando del $26,8 \%$ en el pre-test al 36,4\% en el post-test), del $46 \%$ en el uso siempre del preservativo con pareja ocasional (pasando del 48,2\% en el pre-test al 70,4\% en el post-test) y del $19 \%$ en el porcentaje de relaciones coitales protegidas (pasando del 49,7\% en el pretest al 59\% en el post-test). Estos cambios positivos y significativos, salvo en el uso del preservativo con pareja ocasional, se mantienen en el seguimiento, donde se observa un aumento del $21 \%$ en el porcen- taje de relaciones coitales protegidas en relación al pre-test y un aumento del 38\% en el uso siempre del preservativo con pareja habitual. Estos resultados apoyan la eficacia de este tipo de talleres para aumentar la frecuencia de las conductas sexuales protegidas entre los sujetos que participen en ellos. El retorno, en el seguimiento, del uso "siempre" del preservativo con pareja ocasional a niveles inferiores al pre-test, nos hace pensar en un grupo de sujetos de alto riesgo y más reticentes al cambio que será objeto de una valoración específica en un próximo trabajo. En este estudio utilizamos un criterio restrictivo como indicador de resultados (el uso "siempre" del preservativo en las relaciones coitales), debido a que es un objetivo de los TSMSs, que queríamos poner a prueba, sabiendo que también el uso "a veces" del preservativo con pareja ocasional es preventivo, especialmente cuando este uso se produce en las relaciones con parejas ocasionales de mayor riesgo (Pinkerton y Abramson, 1997).

Algunos autores (Weeks y cols., 1995) consideran dos tipos de autoeficacia en el contexto de la prevención de la transmisión del VIH/SIDA: la autoeficacia de uso (habilidad para conseguir y utilizar medidas preventivas como usar el preservativo) y autoeficacia de rechazo (la que se requiere para no dejarse inducir por proposiciones para llevar a cabo conductas de riesgo como la relación sexual sin protección). Los resultados relativos a los problemas con el preservativo (problemas para convencer y problemas con el manejo y uso del preservativo) están directamente relacionados con estos dos tipos de autoeficacia y demuestran una clara mejoría entre los usuarios de los TSMSs tras los talleres que se mantiene en el seguimiento, suministrando apoyo adicional a su eficacia. De hecho, el entrenamiento en las habilidades para el manejo y uso adecuado del preservativo y las habilidades para una comunicación interpersonal, negociación y asertividad, constituyeron un elemento clave de estos talleres, por lo que es de esperar que disminuyan sustancialmente este tipo de dificultades entre los usuarios de los TSMSs.

Como una limitación de nuestro estudio, tenemos que señalar que la asignación de los sujetos al grupo de intervención o al grupo de comparación no se ha realizado de forma aleatoria sino de acuerdo con necesidades clínicas, de modo que el diseño de la evaluación es cuasi-experimental con grupos de control no equivalentes. No obstante, los resultados han demostrado que los usuarios de los TSMSs eran globalmente muy similares (en el pre-test) a los sujetos de los grupos de comparación en las variables utilizadas como indicadores de los resultados. Esta equivalencia aumenta la validez interna de las atribuciones de los cambios en el post-test al impacto de los TSMSs (Alvira, 1991). La mayoría de los cambios favorables que se observan entre los usuarios de los TSMSs a lo 
largo del estudio se producen en el post-test en relación al pre-test, lo que proporciona validez interna a estos talleres como causa de la reducción de las conductas sexuales de riesgo y de la mejora en las variables mediadoras de estas conductas.

Sin embargo, nos encontramos con un efecto que limita notablemente la posibilidad de atribuir los resultados positivos observados exclusivamente a las intervenciones desarrolladas en los TSMSs: los sujetos de comparación también parecen modificar sustancialmente algunos indicadores conductuales de resultados en relación con el pre-test; como son el uso del preservativo con la pareja habitual, el porcentaje de relaciones coitales protegidas y los problemas experimentados con el preservativo. Atribuimos estos resultados positivos e inesperados en el grupo de comparación (al menos parcialmente) a un efecto del modelado debido al aprendizaje social, puesto que estos sujetos perciben por reforzamiento vicario que es normativo realizar conductas de protección en su grupo de referencia en un momento determinado (durante la realización de los TSMSs). Los grupos de comparación están formados por sujetos admitidos a tratamiento en los mismos programas que los usuarios de los TSMSs. Es bien sabido que estos grupos de usuarios de drogas en tratamiento son altamente endogámicos en su red social (incluso en el ámbito de las relaciones íntimas) y que existe un alto nivel de interacción entre ellos, dentro y fuera de los contextos del tratamiento. Por este motivo, un programa de nueva implantación como es un TSMS puede tener un impacto por modelado social que va más allá de su efecto directo sobre los usuarios (sin olvidar que los sujetos de comparación fueron informados, a través de las instrucciones para su participación en el estudio, de la existencia de un programa para la reducción de los riesgos asociados a la conducta sexual). Diversos estudios señalan que la aprobación por parte de personas afectivamente vinculadas (parejas sexuales o de consumo) es muy determinante para la adopción y modificación de conductas de riesgo entre UDIs, (Perí, Saltó y Bayés, 1994), que la modificación de estos comportamientos sexuales de riesgo es más probable cuando es reforzada socialmente, en lugar de recibir el rechazo de las personas de referencia (Des Jarlais y Friedman, 1988) y que las redes sociales de los UDIs rechazan más fácilmente los comportamientos de riesgo asociados al consumo de drogas que las conductas sexuales de riesgo (llegando incluso a aprobarlas), lo que podría explicar también la mayor resistencia al cambio en estas últimas (Rhodes, 1997). Esta atribución (al menos parcial) del cambio positivo en los sujetos de comparación al posible efecto del modelado social encuentra apoyo en otros dos efectos observados en nuestro estudio: los cambios positivos observados en los sujetos de comparación no se observan en las variables mediadoras que expli- carían estos cambios comportamentales (conocimientos sobre el preservativo, sobre las situaciones de riesgo y medidas de protección frente a la transmisión del VIH/SIDA y percepción de las ventajas del preservativo), como ocurre entre los usuarios de los TSMSs. Únicamente se observan cambios en sentido positivo entre los sujetos de comparación en la reducción de variables mediadoras que indican "trabas" para el uso del preservativo, y que resultan más relevantes una vez que el sujeto ha decidido usarlo: expectativas de resultados negativos del preservativo y desventajas asociadas al mismo. Además, los cambios positivos en las conductas de protección entre los sujetos de comparación se producen más entre el post-test y el pre-test (un periodo de dos semanas o menos) que entre el pre-test y el seguimiento (un periodo de un mes y medio), coincidiendo con los cambios en los usuarios de los TSMSs.

En conclusión, nuestra interpretación de los resultados observados consiste en atribuir los resultados positivos observados en las conductas de protección, en los problemas experimentados con el preservativo y en las variables mediadoras entre los usuarios de los TSMSs al efecto de estos talleres y atribuir en cierta medida (y a modo de hipótesis) los efectos positivos observados en las conductas de los sujetos de comparación al modelado social por reforzamiento vicario de tales conductas de protección. Este efecto de modelado social para la modificación de conductas de riesgo resistentes al cambio supondría un apoyo adicional a la eficacia de las intervenciones breves (Talleres) orientadas a la reducción de riesgos para la transmisión de enfermedades infecto-contagiosas relacionadas con la conducta sexual, que se añadiría a la reconocida eficiencia de las intervenciones grupales con usuarios de drogas en tratamiento.

Por último, hay que tener en cuenta que si bien el seguimiento se realizó al mes de la finalización de los talleres para evitar la mortandad experimental característica de este colectivo, que hubiera eliminado de la muestra a muchos talleres; somos conscientes de la necesidad de realizar seguimientos a más largo plazo para valorar la evolución de los cambios obtenidos.

En conclusión, podemos decir que los resultados hallados proporcionan una evidencia consistente de apoyo a la eficacia de los TSMSs para el logro de sus objetivos a corto y a medio plazo y una elevada satisfacción de los usuarios de drogas que participan en ellos, demostrando la importancia que tiene la puesta en marcha de este tipo de programas de reducción de riesgos en los servicios que trabajan con usuarios de drogas. Asimismo, demuestra que la red de profesionales creada a través del Programa de Formación de Formadores ha logrado su objetivo de poner marcha programas eficaces de reducción de los riesgos asociados a la conducta sexual con los usuarios de dro- 
gas, aportando evidencia sobre la eficiencia del trabajo grupal y en redes con profesionales sanitarios.

\section{AGRADECIMIENTOS}

Este trabajo ha sido posible gracias a la colaboración entre el Plan Nacional sobre Drogas, el Plan Nacional sobre el Sida y la Universidad del País Vasco, y al trabajo de los profesionales de las distintas CC.AA que participaron en él. Asimismo, las autoras quieren agradecer a los revisores anónimos del borrador por sus comentarios acertados y pertinentes.

\section{BIBLIOGRAFÍA}

Ajzen, I. y Fishbein, M. (1977). Attitude and Behavior relations: A theoretical analysis and review of empirical research. Psychological Bulletin, 84, 888-918.

Ajzen, I. y Fishbein, M. (1980). Understanding Attitudes and Predicting Social Behavior. USA: Prentice-Hall, Inc.

Ajzen, I. y Madden, T.J. (1986). "Prediction of goal-directed behavior: The role of intention, perceived control, and prior behavior". Journal of Experimental Social Psychology, No 26, 305-328.

Alvira, F. (1991). Metodología de la evaluación de programas. Madrid: Centro de Investigaciones Sociológicas.

Bandura, A. (1982). Social Learning Theory. Englewood Cliffs, NJ: Prentice-Hall, Inc. (Versión Castellana: Teoría del Aprendizaje Social. Madrid: Espasa-Calpe).

Battjes, R.J.; Pickens, R.W. y Brown, L.S.; (1995). "HIV infection and AIDS risk behaviors among injecting drug users entering methadone treatment: an update". Journal Acquired Immune Deficiency Syndrome, $N^{\circ}$ 10, 90-96.

Becker, E.; Rankin, E. y Rickel, A. (1998) High-Risk Sexual Behavior: Interventions with Vulnerable Populations. New York: Plenum Press.

Becker, M.H. (1974). "The health beliefs model and personal health behavior", Health Education Monographs, $N^{\circ}$ 2, 324-473.

Catania, J.A.; Kegeles, S. y Coates, T.; (1990). "Towars an understanding of risk behavior: An Aids risk reduction model (ARRM),"Health Education Quarterly, 17: 53-72.

Corby, N.H.; Schneider, J.M.; Wolitski, R.J.; (1997). Using the theory of planned behavior to predict intention to use condoms among male and female injecting drug users. Journal of Applied Social Psychology, 26: 52-75.

Des Jairlais, D.C.; Friedman, S.; (1988). The psychology of preventing AIDS among intravenous drug users: A social learning conceptualization. American Psychologist, 43, 11: 865-870.

Des Jarlais, D.C.; Friedman, S.; Perlis, T. et al., (1999). “Behavior and HIV infection among new drug injectors in the era of AIDS in New York City", Journal of Acquired Immune Deficiency Syndrome Human Retrovirology, 20: 67-72.

Dolan, K.A.; Stimson, G.V. y Donoghoe, M. (1993). Reductions in HIV risk behavior and stable HIV prevalence in syringeexchange clients and other injectors in England. Drug and Alcohol Review, 12, 133-142.

Dolezal, C.; Meyer-Bahlburg, H.F.L.; Liu, X.H.; Ehrhardt, A.A.; Exner, T.M.; Rabkin, J.G.; Gorman, J.M.; Marder, K.; Stern, Y. (1999). Longitudinal changes in sexual risk behavior among HIV+ and HIV- male injecting drug users. American Journal Of Drug And Alcohol Abuse, 25, 2: 281-303.

Ehrhardt, A.; Exner, T.; Miller, S. y Stein, Z. (1992). Sexual risk behavior and behavior change in heterosexual women and men. New York: Center for Clinical and Behavioral Studies at the New York State Psychiatric Institute.

Falk, R.S.; Wang, J.C.; Carlson, R.G.; Siegal, H.A. (1997). Factors influencing condom use among heterosexual users of injection drugs and crack cocaine. Sexually Transmitted Diseases, 24, 4: 204-210.

Gibbons, F.; McGovern, P. y Lando, H. (1991). "Relapse and risk perception among members of smoking cessation clinic", Health Psychologist, 10: 42-45.

Green, L.W. y Kreuter, M.W. (1991). Health promotion planning: An educational and environmental approach. Mountain View, CA: Mayfield.

Insúa, P. (1996). La Prevención del SIDA a Nivel Comunitario: Un Programa de Investigación-Acción con el Colectivo de Consumidores de Droga por Vía Parenteral. En San Juan, C.; (coord..): Intervención psicosocial: Elementos de Programación y Evaluación Socialmente Eficaces. Colombia: Anthropos., págs. 134-168.

Insúa, P. (1999). Manual de Educación Sanitaria: Recursos para diseñar Talleres de prevención con usuarios de drogas. Madrid: PNSD y UPV/EHU.

Insúa, P. (2002). Evaluación de los Talleres de Sexo Más Seguro y Consumo de Menos Riesgo para Usuarios de Drogas. Características Psicométricas de los Instrumentos de Medida. Documento no publicado. Madrid: PNSD.

Insúa, P.et al.; (1993). Sexo Seguro: Programa de Prevención de la Transmisión Heterosexual del VIH para CDVPs. En: Usieto, R.; De Andrés, R.; Cuberta, F.; (eds.): Transmisión Heterosexual del VIH. Guadalajara: CESA, págs. 81-91.

Insúa, P y Moncada, S. (2000a). "Opinión expresada sobre un Programa de Formación de Formadores para profesionales que trabajan con UDIs", Adicciones, 12, 4: 479-486.

Insúa, P y Moncada, S. (2000b). “ ¿Cómo diseñar un programa de prevención de la transmisión sexual del VIH para usuarios de drogas?". Trastornos Adictivos, 2,3: 201-210. Insúa, P y Moncada, S. (2001). “ ¿Está cambiando el discurso sobre el uso de drogas en las Instituciones?: Un Programa de Formación de Formadores". Revista Española de Drogodependencias, 26, 1: 6-28. 
Insúa, P., Lledó, M y Grijalvo, J. (2001). "Nuevos conceptos, nuevo discurso: Talleres de consumo de menos riesgo". Trastornos Adictivos, 3, 2: 111-120.

Insúa, P. y Moncada, S. (2002). Cognición, Actitud y Conducta: Cambio en UDIs hacia la reducción de los riesgos asociados al uso de drogas. Aceptado para su publicación en Adicciones, 14, 2.

Menoyo, C.; Urzelai, A.; Zubia, I. y Zulaika, D. (1998). “Variación temporal de las conductas de riesgo en relación con la infección por VIH". SEISIDA, 9, 3: 133-135.

Páez, D. et al. (1996). Evaluación del cambio de actitudes y conductas ante el SIDA. En: Basabe, N.; Páez, D.; Usieto, R.; Paicheler, H.; Deschamps, J.C. (Eds.): EI desafío psicosocial del SIDA. Madrid: Fundamentos y CESA, págs. 113-135.

Pavia, M.; Indovino, A.; Nobile, C.; et al. (1997). "Intravenous drug users and AIDS". European Journal Public Health, 7, 2: 199-204.

Perí, J.M.; Saltó, C.; Bayés, R. (1994). Factores facilitadores del cambio de comportamientos de riesgo al $\mathrm{VIH}$ en usuarios de drogas por vía parenteral. Medicina Clínica, 102: 237-238.

Pinkerton, S.D.; Abramson, P.R. (1997). Occasional condom use and HIV risk reduction. Journal of Acquired Immune Deficiency Syndromes and Human Retrovirology. 13 (5): 456-460.

Prochaska, J.O. y DiClemente, C.C. (1983). "Stages and processes of self-change of smoking: Toward and integrative model of change". Journal of Consulting and Clinical Psychology, 51: 390-395.
Prochaska, J.O. y DiClemente, C.C. (1992). "Stages of change in the modification of problem behaviors". En: M. Hersen, R.M. Eisler y P.M. Miller (editores): Progress in behavior modification. Sycamore, Illinois: Sycamore Press, págs. 184-214.

Rhodes, T. (1997). Risk theory in epidemic times: sex, drugs and social organisation of "risk behaviour". Sociology of Health and IIIness, 19, 2: 208-227.

Schifter, D.E. y Ajzen, I. (1985). “Intention, perceived control and weight loss: An aplication of the theory of planned behavior". Journal of Personality and Social Psychology, 49: 843-851.

Stimsom, G.V. y Power, R. (1992). Assessing AIDS prevention for injecting drug users: some methodological considerations. British Journal of Addiction, 87, 3: 455-465.

Stimson, G.V. (1995). AIDS and Injecting Drug Use in the United Kingdom, 1987-1993: The Policy Response and the Prevention of the Epidemic. Social Science Medicine, 41, 5: 699-716.

Watters, J.; Estilo, M.; Kral, A.; et al. (1994). HIV infection among female injection-drug users recruited in community settings. Sexually Transmitted Diseases, 21, 6: 321-328.

Watters, J.; Estilo, M.; Clark, G.L.; Lorvick, J. (1994). Syringe and needle exchange as HIV / AIDS prevention for injection drug users. JAMA, 271, 2: 115-120.

Weeks, K.; Levy, S.R.; Zhu, C.; Perhats, C.; Handler, A.; Flay, B.R. (1995). Impact of a school-based AIDS prevention program on young adolescents' self-efficacy skills. Health Education Research, 10, 3: 329-344. 
